\title{
Infants Actively Construct and Update Their Representations of Physical Events: Evidence from Change Detection by 12-Month-Olds
}

\author{
Su-hua Wang and Elizabeth J. Goldman \\ University of California, Santa Cruz, CA, USA \\ Correspondence should be addressed to Su-hua Wang; suhua@ucsc.edu
}

Received 30 April 2016; Revised 30 September 2016; Accepted 20 October 2016

Academic Editor: Gedeon Deak

Copyright (C) 2016 S.-h. Wang and E. J. Goldman. This is an open access article distributed under the Creative Commons Attribution License, which permits unrestricted use, distribution, and reproduction in any medium, provided the original work is properly cited.

\begin{abstract}
The present research investigates the effects of top-down information on 12-month-olds' representations of physical events, focusing on their ability to detect an object change across different events. Infants this age typically fail to detect height changes in events with tubes even though they successfully do so in events with covers. In Experiment 1, infants who saw a tube event in which objects did not interact successfully detected a change in an object's height, suggesting that object interaction affects infants' categorization of physical events. Experiments 2 and 3 examined the fine-grained process of event representation. In Experiment 2, infants detected the change in the tube event if they were led by pretest exposure to believe that the event was conducted with a cover. In Experiment 3, infants who initially believed so updated their representation if shown a tube before object interaction occurred (but not after). Together, these findings provide new evidence that infants, like older children and adults, actively construct physical events. Whether they notice a change depends on their existing knowledge and the current representation of the event.
\end{abstract}

\section{Introduction}

Children's understanding about the physical world undergoes profound developments in the first two years [1-3]. Past research has documented the emergence of infants' ability to use object information in various types of physical events. Starting from a young age, infants are capable of tracking objects that move in relation to one another, attending to spatial arrangement among objects and anticipating physical events to unfold in a certain manner [4-11]. The emergence of these abilities also comes with limitations, which provide useful test beds for researchers to specify learning mechanisms underlying the early development of physical knowledge. One unique limitation is that infants would fail to use object information in some events despite their success in doing so in other events at the same age. Curious decalages have been observed in infants' ability to use object information across different types of physical events. Infants notice some event outcomes inconsistent with a physical rule but fail to notice other outcomes that violate the same rule until they are much older $[12,13]$.
For example, infants at 3.5 months notice improbable outcomes of occlusion events when tall objects become fully hidden behind short occluders (e.g., [14]). However, until about 7.5 months infants fail to notice improbable outcomes of containment events that violate the same rule of height when tall objects become fully hidden inside short containers (e.g., [12, 13]), and until 12 months they fail to detect improbable outcomes of covering events that violate this rule (e.g., [15]). Thus, events that are inconsistent with the physical rule of height can be detected or go unnoticed depending on the spatial relation involved [16].

A similar discrepancy is observed in infants' ability to notice impossible changes to object properties. When seeing an improbable change in an object's feature during an event, infants' ability to detect the change is related to the type of the event, a factor that should have been independent of the persistence of object feature [17] (for infants' early success in using object features for the purpose of categorization, see, e.g., [18-20]). For example, one study compared infants' ability to notice a change in an object's height across different types of events [21]. The infants first saw a cylindrical 
cover standing next to a short object. An experimenter then rotated the cover upward 90 degrees to show its open end and hollow interior. Next, the cover was lowered over the object. After a pause, the cover was lifted, revealing either the same or a much taller object (a between-subjects design). Eleven-month-old infants who saw this covering event looked equally whether the object changed its height or not, displaying change blindness. Interestingly, when the cover was lowered in front of the object, creating an occlusion relation, 11-month-olds noticed the change, looking reliably longer if shown the change event than the no-change event. Thus, the same change in the object's height can be detected or go unnoticed depending on whether the cover was lowered behind or over the object. More strikingly, when infants at 12.5 months saw events that involved the same spatial relation but used different hiding devices and a cover or a tube was lowered over the object, the infants in the cover condition noticed the change of object height, but those in the tube condition did not.

The findings reviewed so far give rise to a curious question: if infants attend to information about object height as young as 3.5 months in some events, why do they fail to do so in other events until much older? A theoretical account of infants' physical reasoning suggests an answer to this question [22-24]. Below, we summarize the account in terms of three findings and then illustrate how it helps explain the decalage.

First, research has shown that in their first year of life, infants form distinct event categories, such as occlusion, containment, support, and collision events (e.g., [25-28]). For example, a recent report [28] showed that after watching several different examples of containment events, 8-monthold infants treated a new containment event as familiar and a support event as novel, suggesting that they formed an abstract category of containment and distinguished it from support. At the age of 11 months, infants provided evidence that they formed an abstract category of containment and distinguished it from occlusion. Each of these event categories represents a type of causal interaction in which objects serve distinct roles (e.g., occluder, occluded). The type of interaction is determined primarily by the spatiotemporal relation between objects and by certain object properties. For example, whether infants consider an event as occlusion or covering depends on whether the event involves an object behind or under the hiding device, and whether it is considered as a covering or tube event depends on whether the hiding device features one or two open ends $[15,21,29]$.

Second, for each event category, infants gradually learn about the information relevant to predicting event outcomes (e.g., [12, 24, 30]). For example, at about 3 months, infants learn that whether an occluder has a continuous lower edge determines whether it will hide an object from view. When an object passes behind an occluder whose lower edge is not continuous but instead creates an opening between the occluder and the surface on which it stands, infants expect the object to appear in this opening; when no such opening exists, infants expect the object to remain hidden $[4,31]$. At about 3.5 months, infants further learn that the height of the occluder relative to that of the object determines the outcome of occlusion events. Putting the two pieces of information together, they now recognize that if an object is taller than any portion of an occluder with a continuous lower edge, the object will still be partly visible when passing behind the occluder [12-14].

Third, research has shown that in the first two years, infants often fail to generalize what they learn in the context of one event category to the context of another category $[16,32,33]$. For example, infants who learned, through deliberate experience in the laboratory, that height is relevant to covering events failed to use height information when watching a tube event [29].

Linking the above findings, a theoretical account has been proposed suggesting that infants' representation of objects in a physical event is influenced by how they categorize the event, what they have learned so far about this category of events (e.g., what information is relevant to this type of events), and their limited transfer of learning across different event contexts in the first two years [22-24]. The account helps explain the surprising age gap in infants' ability to notice implausible outcomes or implausible changes to an object across different types of events. However, the exact mechanism underlying these phenomena remains unclear. For example, do infants bring to bear the categorical cues when watching all kinds of physical events, and what constitutes the critical component that triggers the effects of event categories? Moreover, how do infants incorporate various cues that they receive at different points in time during an event? Guided by these questions, the present research sought to clarify the mechanism underlying infants' representations of physical events by testing the following three predictions.

First, prior findings of event categorization have all involved infants' observation of multiple objects interacting in time and space, which leads us to predict that object interaction plays a crucial role in triggering the effects of event categories. Evidence that infants' use of object information is no longer affected by event categories when object interaction is removed from the event will support this prediction (Experiment 1). Second, in the existing work, information that guides infants to select a category for the current event (henceforth, categorical cues) has all been shown during the event, making it necessary to compare infants' use of object information when they watch perceptually different events. However, the account described above predicts that if infants receive categorical cues before the event, they should still be led to select the corresponding category during the event; thus, their success or failure to use information during the event will still depend on the cues provided beforehand. Evidence that infants' use of object information in perceptually identical events differs depending on the cues they receive beforehand will support this prediction (Experiment 2). Third, categorical cues shown to infants after object interaction have taken place should cease to affect infants' use of object information. Evidence that infants' use of object information varies depending on the timing of the categorical cue will support this prediction (Experiment 3). Testing these predictions will shed light on the origin of the decalages reported in the existing work; more broadly, the findings will help delineate the fine-grained aspect of the process by which infants represent objects in physical events. 
In all of the following experiments, half of the infants within each condition saw a change event in which an object changed its height, and half saw a no-change event. We compared the two groups' looking times to examine whether infants who saw the change event looked significantly longer than those who saw the no-change event, a response pattern that would indicate change detection. Testing whether infants detect a change in an object has been utilized as a straightforward method to clarify whether infants have represented and used object information [24]. Similar to the prior work [21], the between-subjects design was chosen in the present research to avoid the possibility that watching the change event (potentially surprising to infants) could have exerted influence on their response to the subsequent, no-change event.

\section{Experiment 1}

Under what conditions do infants attend to and represent information about object features? A report showed that infants who had just failed to detect a change in an object's height during a covering event were able to distinguish between the pre- and postchange objects from an object they had not seen before by height, if the objects were presented in a static display [34]. In the display, objects did not move at all and did not interact with one another. This finding lends support to our hypothesis that object interaction may be critical in triggering the effects of event categories on infants' use of object information. Alternatively, it could be that any object movement can trigger the effects, including the case when objects move side by side without interaction. Experiment 1 attempted to tease apart these possibilities and tested the prediction that interaction, not movement, plays a crucial role in the process.

Specifically, we tested the prediction using tube eventsevents that involve a tube and other objects. Research has shown that infants younger than 14 months typically fail to notice implausible outcomes or changes involving height when they watch tube events $[15,21,29]$. In the present experiment, 12-month-old infants watched a tube event in which an object that stood next to a tube either changed or maintained its height over the course of the test trial. The event was similar to the tube event in the previous research [21] with a crucial modification: the infants in the present experiment were turned away from the apparatus during object-tube interaction when the tube was lowered over the object, fully hiding it, and then lifted to reveal the object (see Figure 1 for the event segments that the infants did watch). Turning infants away, rather than lowering the apparatus curtain or showing a different event, ensured that infants' processing of the test event was not interfered by exposure to another event. At the end of the trial, the object either remained the same or changed from short to tall. Another group of 12-month-olds were tested in a similar way, except that the change was from tall to short.

If object interaction plays a crucial role in triggering the effects of event categories on infants' use of object information, removing object interaction should prevent the 12-month-olds from being affected. The infants could now rely on their memory of object features, making it more likely for them to detect the change (given that even 5- or 6month-olds have no difficulty retaining height information for several seconds [35-37]). As a result, infants in both conditions (short-to-tall or tall-to-short) should look reliably longer if they were shown a change event than if shown a nochange event.

\subsection{Method}

2.1.1. Participants. Participants were 32 healthy term infants (range: 11 months 3 days to 12 months 23 days; 17 boys and 15 girls). Half of the infants were randomly assigned to the shortto-tall condition ( $M=11$ months 28 days) and half to the tall-to-short condition ( $M=12$ months 4 days). Additional four infants were tested but excluded from analysis, due to fussiness $(n=1)$ or distraction $(n=1)$ or because the observers could not follow the infant's eye gaze $(n=2)$. In this and the following experiments, participants were primarily Caucasian from middle-class backgrounds and recruited from birth announcements and local hospitals. Parents received travel reimbursements but were not otherwise compensated for their participation.

2.1.2. Apparatus. The apparatus was the display box $(106 \mathrm{~cm}$ high, $103.5 \mathrm{~cm}$ wide, and $60 \mathrm{~cm}$ deep). Infants faced a large opening in the front of the box. Prior to the test trial, a curtain was lowered in front of the opening, fully concealing it. The stimuli were identical to those in [21], consisting of a blue cylindrical cover or tube $(11.5 \mathrm{~cm}$ in diameter, $16.5 \mathrm{~cm}$ tall) and a red rectangular object ( $5.5 \mathrm{~cm}$ wide, $5.5 \mathrm{~cm}$ deep). The object protruded through a hole in the apparatus floor and was attached to a platform under the floor. During the change event, the platform was raised to extend the visible portion of the object, changing it from short $(4.5 \mathrm{~cm})$ to tall $(15.5 \mathrm{~cm})$ or from tall to short.

2.1.3. Procedure. Each infant sat on a parent's lap in front of the apparatus, about $60 \mathrm{~cm}$ from the lowered curtain. The parent sat on an adjustable-height swivel chair; the experimenter ensured the infant's eye level to be about $8 \mathrm{~cm}$ above the apparatus floor so that the infant could not see the top of the cover or tube. Parents were instructed to remain quiet and keep their eyes closed during the test trial.

No pretest showing was administered. The infants received a single test trial in which they watched a no-change or a change event. At the beginning of the trial, the infants saw a static display of the tube and the object (Figure 1, Box (1) in each row). After the infants had looked at the display for two cumulative seconds, the experimenter grasped the tube $(2 \mathrm{~s})$ and rotated it ( $2 \mathrm{~s}$; Figure 1, Box (2) in each row). After the infants had looked at this scene for two cumulative seconds, the tube was rotated back $(2 \mathrm{~s})$ to its upright position again, $2.5 \mathrm{~cm}$ above and next to the object (Figure 1, Box (3) in each row). Next, the infants were turned away to face a white wall. During the next 10 seconds while the infants faced the wall the tube was moved to the right (1 s), lowered over the object 
Experiment 1

Short-to-tall condition

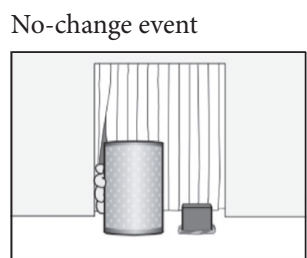

(1) Static display

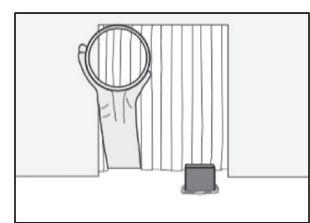

(2) Rotate tube

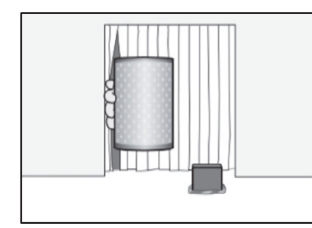

(3) Lift tube

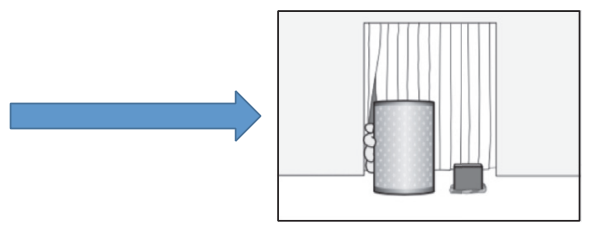

(4) Infant away from apparatus

Change event

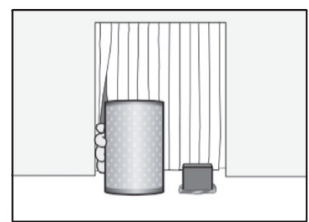

(1) Static display

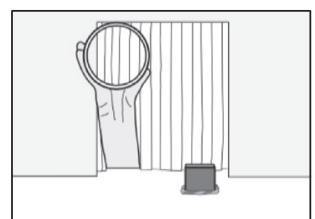

(2) Rotate tube

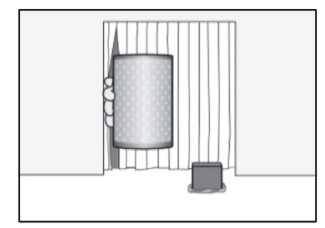

(3) Lift tube

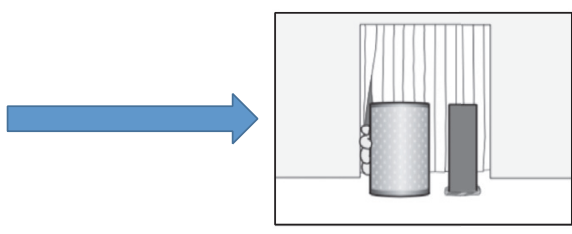

(4) Infant away from apparatus
(5) Return tube

Tall-to-short condition

No-change event

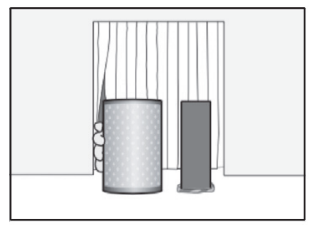

(1) Static display

Change event

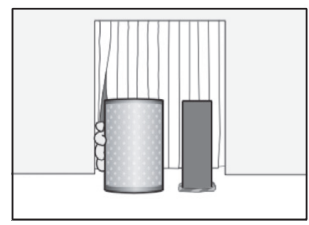

(1) Static display

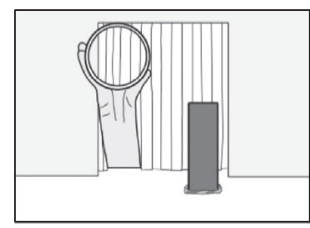

(2) Rotate tube

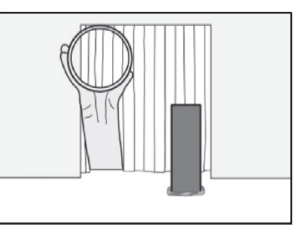

(2) Rotate tube

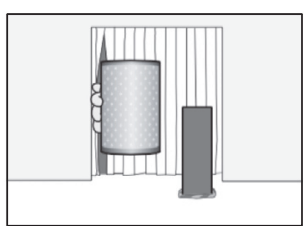

(3) Lift tube

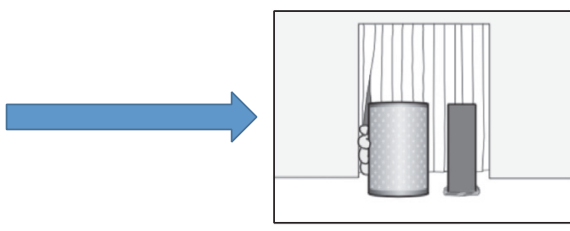

(4) Infant away from apparatus
(5) Return tube

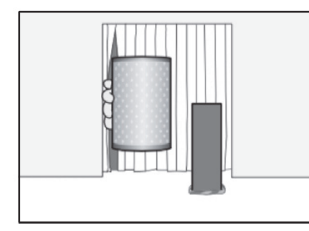

(3) Lift tube

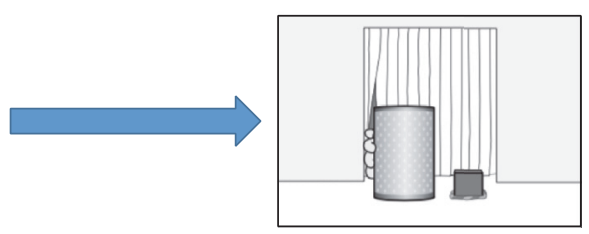

(4) Infant away from apparatus

(5) Return tube

FIGURE 1: Test events shown to infants in Experiment 1. The event sequence and timing were the same as in [21], except that infants were prevented from seeing object interaction.

( $2 \mathrm{~s})$, and released ( $1 \mathrm{~s})$; after a pause (1s), the experimenter grasped the tube $(2 \mathrm{~s})$, lifted it $(2 \mathrm{~s})$, and moved it to the left (1s). Finally, the infants were returned to the initial position and saw the tube being lowered to its starting position next to either the unchanged or changed object ( $2 \mathrm{~s}$; Figure 1, the last box in each row).

Prior to the experiment, the parents sat on the swivel chair and practiced turning around 180 degrees and back. They were instructed to turn the infants 180 degrees, away from the apparatus to face the white wall, upon hearing the first signal, and to turn the infants back facing the apparatus at the second signal. Throughout the trial, observers continuously monitored the infants' gaze. None of the infants looked at the event area during the $10 \mathrm{~s}$ interval. Two observers watched the infants through peepholes in cloth-covered frames on either side of the apparatus. Each observer pressed a button on a controller linked to a computer running windowsbased software when the infant looked at the scene. The observers were not aware of the condition and event assigned to the infant. As in the previous research [21], the trial ended when infants looked away from the final paused scene for 2 consecutive seconds after having looked at it for at least 3 cumulative seconds or when they had looked at the scene for 60 cumulative seconds. These criteria were used for all of the experiments in the current study.

Reported looking times were based on input from the primary (typically more experienced) observer. To assess interobserver agreement, infants' looking times at the final 
TABLE 1: Mean looking times (in seconds) and standard deviations (in parentheses) at the change and no-change events, separately per experiment and condition.

\begin{tabular}{|c|c|c|c|c|}
\hline \multicolumn{5}{|c|}{ Event } \\
\hline & Change & No-change & & Cohen's \\
\hline & $M(\mathrm{SD})$ & $M(\mathrm{SD})$ & $p$ & $d$ \\
\hline \multicolumn{5}{|l|}{ Experiment 1} \\
\hline Short-to-tall condition & $35.0(12.9)$ & $17.7(10.5)$ & $0.007^{*}$ & 1.48 \\
\hline Tall-to-short condition & $33.6(15.9)$ & $15.8(6.6)$ & $0.006^{*}$ & 1.46 \\
\hline \multicolumn{5}{|l|}{ Experiment 2} \\
\hline Show-cover condition & $34.0(18.7)$ & $15.8(7.2)$ & $0.004^{*}$ & 1.28 \\
\hline Show-tube condition & $16.2(6.0)$ & $17.1(5.9)$ & 0.825 & -0.16 \\
\hline \multicolumn{5}{|l|}{ Experiment 3} \\
\hline Postinteraction condition & $29.7(16.1)$ & $8.7(3.9)$ & $0.002^{*}$ & 1.79 \\
\hline Preinteraction condition & $18.5(10.3)$ & $22.5(13.8)$ & 0.508 & -0.33 \\
\hline
\end{tabular}

Note. ${ }^{*} p<0.05$.

paused scene of each trial were divided into $100 \mathrm{~ms}$ intervals. Percent agreement was calculated by dividing the number of intervals in which the observers agreed whether the infant was looking at the scene or not by the total number of intervals during this portion of the trial. For 30 infants (only one observer was present for two infants), the agreement averaged $94 \%$ per infant and ranged from $81 \%$ to $99 \%$. Cohen's kappa was also calculated and yielded substantial agreement between the two observers, $\kappa=0.786$.

Preliminary analyses in this and the following experiments revealed no significant effects involving sex, all $p_{\mathrm{s}}>$ 0.10 . Therefore, data were collapsed across sex in subsequent analyses.

2.2. Results and Discussion. The infants' looking times at the final paused scene (Table 1 ) were compared by a $2 \times 2$ analysis of variance (ANOVA), with condition (short-to-tall or tall-toshort) and event (change or no-change) as between-subjects factors. The analysis only yielded a significant main effect of event, $F(1,28)=17.24, p<0.001$. Planned comparisons indicated that infants looked reliably longer if shown the change event than if shown the no-change event; this was the case in the short-to-tall condition, $F(1,28)=8.43, p=$ 0.007 , and the tall-to-short condition, $F(1,28)=8.86, p=$ 0.006 . Nonparametric Wilcoxon Rank-Sum tests confirmed the results (short-to-tall: $W=46, p<0.05$; tall-to-short: $W=$ 42.5, $p<0.025)$.

Inspection of infants' looking times at the test event indicated that being turned away and back did not make the infants disengaged from the remaining test event. All infants looked at the event almost continuously ( $M=95 \%$ of the time) after being turned back to face the apparatus.

Prior research using looking-time and action tasks has converged to show that until infants are about 14 months, they typically fail to notice height violations in tube events $[15,29]$. In Experiment 1, however, the 12-month-olds detected the implausible change in the object's height when they saw a tube being moved next to the object. The key difference between the present experiment and previous studies was that infants in the current experiment did not see the tube being lowered over the object as they were turned away from the apparatus during the segment of object interaction. Thus, the results support our prediction that object interaction plays a critical role in the event-category effects on infants' use of object information. Removing object interaction from the event can prevent infants from being affected by the categorical cues. In the present case, the removal of object interaction facilitated 12-month-olds' change detection because it lifted the constraint that infants this age typically have-the notion that they have not yet learned about the relevance of height information in tube events.

\section{Experiment 2}

Experiment 2 tested the prediction that categorical cues provided prior to the event will guide infants to perceive events differently depending on the cues, even if the events unfold in a perceptually identical manner. Twelve-monthold infants were assigned to a show-cover or a show-tube condition and watched a single test event (Figure 2). The key manipulation was that, different from [21], the hiding device (the cover or the tube) was never rotated during the event in the present experiment. Instead, it was shown to infants before the test trial. During the test trial, infants were seated at an eye level too low to see the top of the device and could not discern whether the device had a closed top (cover) or an open top (tube). Therefore, no categorical cue was provided during the test event, making the event perceptually identical across conditions.

Previous studies show that at 12 months, infants typically fail to use height information in tube events but will succeed in doing so in covering events $[21,29]$. If infants selected an event category based on the device they were shown before the test trial, the infants in the show-cover condition should detect the change and look longer if shown the change event than if shown the no-change event, whereas those in the show-tube condition should miss the change and look equally whether they saw the change or no-change event. 
Experiment 2

No-change event

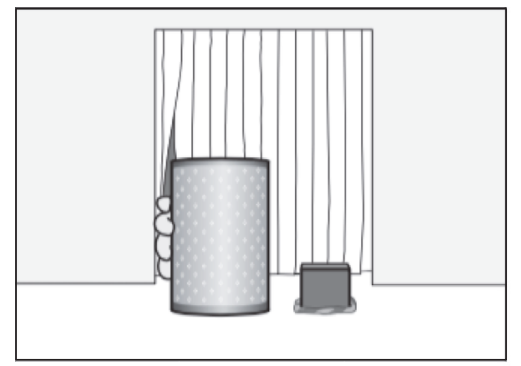

(1) Static display

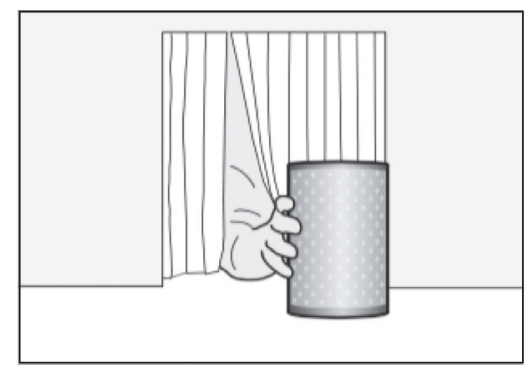

(2) Lower tube over object

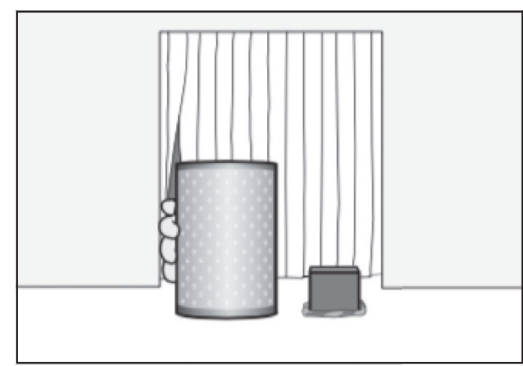

(3) Return tube

Change event

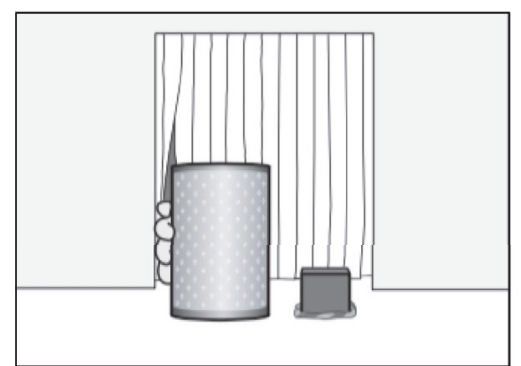

(1) Static display

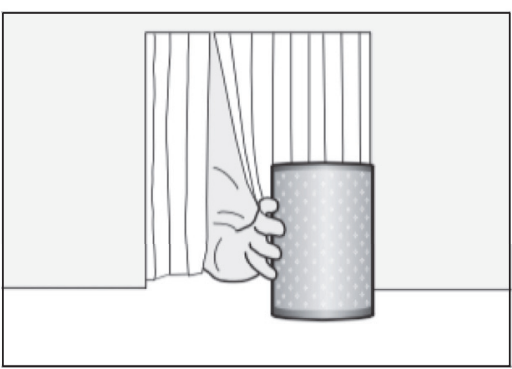

(2) Lower tube over object

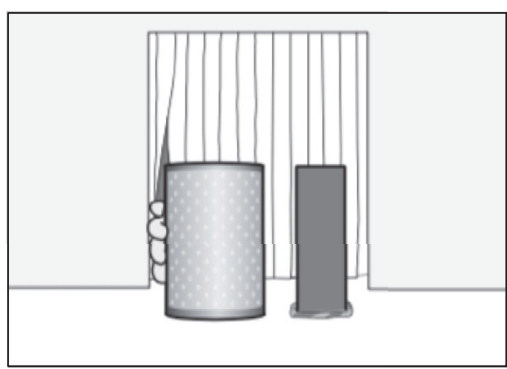

(3) Return tube

Figure 2: Test events shown in the show-cover and show-tube conditions of Experiment 2. The experimenter showed infants a cover or a tube before the test event began.

\subsection{Method}

3.1.1. Participants. Participants were 32 healthy term infants, ranging from 11 months 24 days to 13 months 14 days (14 boys and 18 girls). Half of the infants were randomly assigned to the show-cover condition ( $M=12$ months 18 days) and half to the show-tube condition ( $M=12$ months 22 days). One additional infant was tested but was eliminated due to parental interference.

3.1.2. Procedure. As in Experiment 1, after the infants were seated, the experimenter ensured that their eye level was about $8 \mathrm{~cm}$ above the apparatus floor, too low for them to see the top of the hiding device during the test event. The pretest showing took place in front of the lowered curtain before the test trial began. The experimenter showed each infant the device (the cover or tube) in its upright position for about 2 seconds and rotated it upward 90 degrees to show one or two open ends. The pretest showing ended when the infants made eye contact with the rotated device for 2 seconds (determined by a timer). The experimenter then left with the device. About 15 seconds later, the curtain of the apparatus was raised to start the test trial in which the infants watched either a no-change or a change event.

At the start of the event, the object stood $2.5 \mathrm{~cm}$ next to the hiding device; recall that the infants' position was too low to discern whether the device was a cover or a tube. After the infants had looked at the static display (Figure 2, Box (1) in each row) for two cumulative seconds, the experimenter grasped the device ( $2 \mathrm{~s})$, lifted it (2s), and moved it to the right (1 s) until it was centered about $2.5 \mathrm{~cm}$ above the object. Next, the device was lowered over the object $(2 \mathrm{~s})$, fully hiding it (Figure 2, Box (2) in each row). The experimenter then released the device (1s) and paused (1s). Finally, the experimenter grasped the device again (2s), lifted it (2s), moved it to the left ( $1 \mathrm{~s})$, and lowered it to its starting position ( $2 \mathrm{~s}$; Figure 2, Box (3) in each row). At the end of the event, the object was either the same as or much taller than before. The infants watched the final paused scene until the trial ended; the criteria for ending the trial were the same as in Experiment 1. Interobserver percent agreement was calculated for 29 infants (only one observer was present for 3 infants), which averaged $95 \%$ per infant and ranged from $86 \%$ to $100 \%$. Cohen's kappa indicated high agreement between the observers, $\kappa=0.820$.

In each condition, the cover was used to produce the event for half of the infants, and the tube was used for half of the infants. This design served as manipulation check to ensure that the infants could not judge whether a cover or tube was used during the event. If the infants relied on pretest showing alone to select the category, the actual device used during the event should yield no effect on the results.

3.2. Results and Discussion. The infants' looking times at the final paused scene (Table 1) were compared by a $2 \times$ $2 \times 2$ ANOVA, with condition (show-cover or show-tube), event (change or no-change), and device used during the event (cover or tube) as between-subjects factors. The analysis 
yielded a significant main effect of event, $F(1,24)=4.46, p<$ 0.05 , and a significant Condition $\times$ Event interaction, $F(1,24)$ $=5.49, p<0.05$. Planned comparisons indicated that in the show-cover condition, infants who saw the change event $(M$ $=34.0)$ looked reliably longer than those who saw the nochange event $\operatorname{did}(M=15.8), F(1,24)=9.93, p=0.004$; in the show-tube condition, infants looked equally at the two events (change: $M=16.2$; no-change: $M=17.1), F(1,24)<$ 1. Nonparametric Wilcoxon Rank-Sum tests confirmed these results (show-cover: $W=47, p<0.05$; show-tube: $W=$ $64, p>0.10)$. Note that whether a cover or tube was used during the event did not yield any significant effects (all $F_{\mathrm{s}}<1$ ), confirming that the infants relied on the information provided during pretest showing to select the event category.

When the cue provided prior to the test trial suggested to infants that a cover was used to hide an object during the event, infants succeeded in detecting a change in the object's height. In contrast, when it suggested that a tube was used, infants failed to notice the change. The positive results in the show-cover condition could not stem from infants' intrinsic preference for the tall object at the end state of the change event. If it were the case, the same results should have been obtained in the show-tube condition. Thus, these results confirmed the previous findings $[15,21$, 29], showing that 12 -month-old infants notice implausible events involving height when watching covering but not tube events. Critically, the present results provide evidence for event-category effects when infants watch perceptually identical events. The cue provided beforehand leads infants to select an event category; whether or not they detect a perceptual change to objects depends on which category is selected. More broadly, the results provide evidence that categorical cues can serve as top-down information to affect infants' perception of physical events, demonstrating that infants actively construct events they observe. Categorical cues can guide infants to view perceptually identical events as different.

\section{Experiment 3}

In Experiment 2, the way infants perceived and represented an event was affected by the information provided prior to the event. Experiment 3 further investigated the issue of timing. Specifically, it asks whether new information that overrides initial information about the event category will affect infants' representation of the event and whether the influence of new information will depend on its timing.

Like in Experiment 2, 12-month-olds were shown a categorical cue prior to an event. However, during the event, they received another cue suggesting that the currently selected event type was inaccurate and a different category should have been chosen. The questions of interest were (a) whether infants would update their representation of the event based on new information and (b) whether the timing of new information mattered. Experiment 1 showed that object interaction is crucial for classifying events; thus, we predicted that categorical cues provided after object interaction should cease to influence event categorization and therefore should not influence infants' use of object information.

The infants were shown a cover before the test trial began. However, during the test event when the device was rotated, the infants saw that it was in fact a tube. Based on the results of Experiment 2, seeing a cover beforehand should guide infants to determine the event as covering. If they ignored new information and continued to process the event as such, the infants should succeed in detecting the change. However, if the infants updated the category based on new information, they should view the event as a tube event and fail to detect the change.

To examine the issue of timing, new information was revealed either before or after object interaction. Specifically, it refers to the segment of the event in which the tube was lowered over and then lifted above the object. In a postinteraction condition, the tube was rotated near the end of the event after it had been lifted to reveal the object (Figure 3, Box (3) in the top two rows); in a preinteraction condition, the tube was rotated before it was lowered over the object (Figure 3, Box (2) in the bottom two rows).

\subsection{Method}

4.1.1. Participants. Thirty-two healthy term infants (range: 11 months 25 days to 13 months 22 days; 15 boys and 17 girls) participated. Half were randomly assigned to the postinteraction condition ( $M=12$ months 20 days) and half to the preinteraction condition ( $M=12$ months 16 days). Additional two infants were tested but were eliminated due to inattentiveness or distraction.

4.1.2. Procedure. The procedure was the same as the showcover condition of Experiment 2 with the following modification. In the postinteraction condition, after the device was lowered over the object and then lifted to reveal the unchanged or changed object (object interaction, Table 2), the device was rotated upward 90 degrees $(2 \mathrm{~s})$, showing that it was a tube. After the infants had watched this scene for two cumulative seconds, the tube was lowered onto its starting position. In the preinteraction condition, the rotation of the tube was inserted near the beginning of the event. After the infants had looked at the static display, the experimenter rotated the tube upward 90 degrees. When the infants had watched this scene for two cumulative seconds, the event proceeded as in Experiment 2, with the tube being lifted and lowered over the object. Thus, the lengths of the test events were the same across conditions. Interobserver agreement calculated for all infants averaged 93\% and ranged from $84 \%$ to $100 \%$; Cohen's kappa also indicated substantial agreement between the two observers, $\kappa=0.768$.

4.2. Results and Discussion. The infants' looking times at the final paused scene (Table 1 ) were compared by a $2 \times 2$ ANOVA with condition (post- or preinteraction) and event (change or no-change) as between-subjects factors. The analysis yielded a significant Condition $\times$ Event interaction, $F(1,28)=8.78$, $p<0.01$. Planned comparisons indicated that (a) in the 
Experiment 3

Postinteraction condition

No-change event

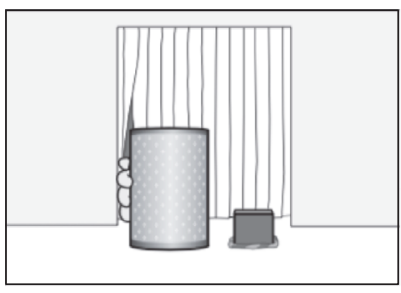

(1) Static display

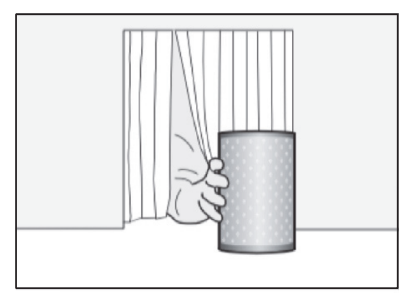

(2) Lower tube over object

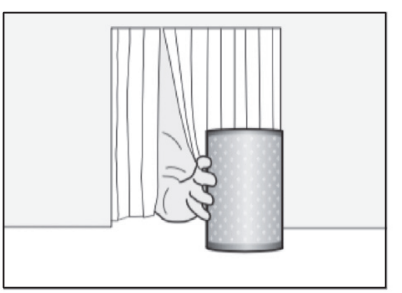

(2) Lower tube over object

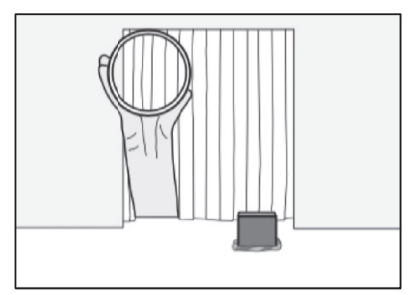

(3) Rotate tube

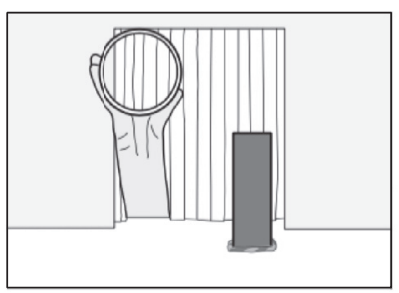

(3) Rotate tube

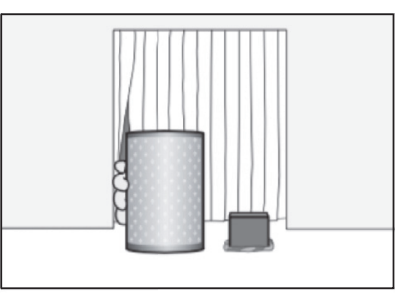

(4) Return tube

Change event

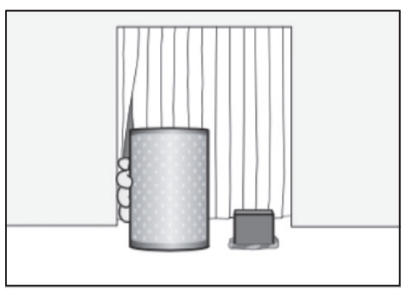

(1) Static display

No-change event

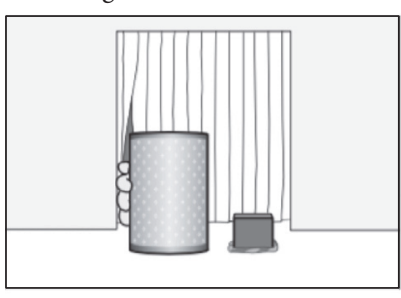

(1) Static display

\section{Preinteraction condition}

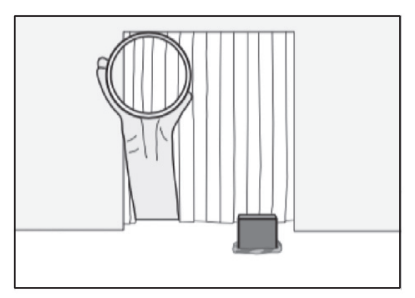

(2) Lower tube over object

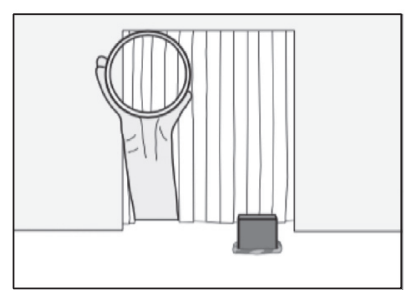

(2) Lower tube over object

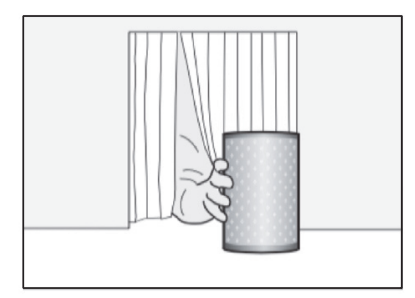

(3) Rotate tube

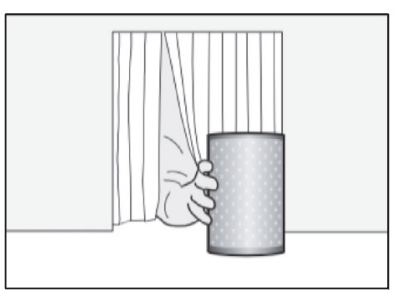

(3) Rotate tube

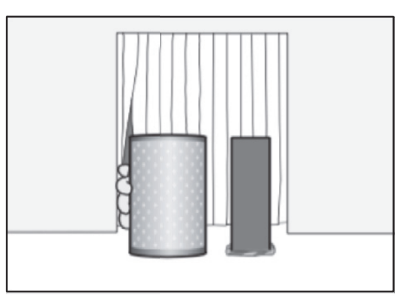

(4) Return tube

Change event

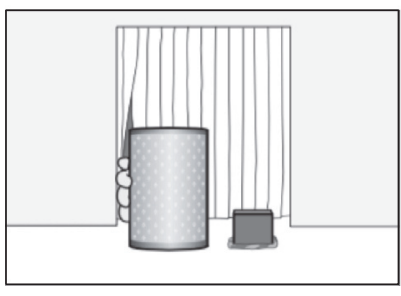

(1) Static display

FIGURE 3: Test events shown in Experiment 3. The two conditions were identical except for the timing of rotating the tube.

TABLE 2: The sequences of event segments involved in Experiments 1 to 3. " $\checkmark$ " indicates that the segment was carried out in the experiment.

\begin{tabular}{|c|c|c|c|c|c|}
\hline & Static display & Rotate tube & $\begin{array}{c}\text { Object interaction: } \\
\text { lower tube over object }\end{array}$ & Rotate tube & Return tube \\
\hline \multicolumn{6}{|l|}{ Experiment 1: } \\
\hline No interaction & $\checkmark$ & $\checkmark$ & & & $\checkmark$ \\
\hline \multicolumn{6}{|l|}{ Experiment 2: } \\
\hline show tube/cover & $\checkmark$ & & $\checkmark$ & & $\checkmark$ \\
\hline \multicolumn{6}{|l|}{ Experiment 3: } \\
\hline Postinteraction & $\checkmark$ & & $\checkmark$ & $\checkmark$ & $\checkmark$ \\
\hline Preinteraction & $\checkmark$ & $\checkmark$ & $\checkmark$ & & $\checkmark$ \\
\hline
\end{tabular}

Note. In Experiment 2, half of the infants were shown a tube and half were shown a cover before the test trial. During the trial, the infants could not see the top of the hiding device. 
postinteraction condition, the infants who saw the change event $(M=29.7)$ looked reliably longer than those who saw the no-change event $\operatorname{did}(M=8.7), F(1,28)=12.40, p=$ 0.002 , and (b) in the preinteraction condition, the infants tended to look equally at the two events (change: $M=18.5$; no-change: $M=22.5), F(1,28)=0.45$. Wilcoxon Rank-Sum tests confirmed these results (postinteraction: $W=40, p<$ 0.005; preinteraction: $W=65, p>0.10$ ).

The infants saw a cover prior to the test trial; during the test event, they saw that the hiding device was indeed a tube. When this new information was provided after object interaction, the infants still succeeded in detecting the change, suggesting that they ignored the information and represented the event according to the initial category (covering events). In contrast, when this new information was provided before object interaction, the infants failed to notice the change, suggesting that they had updated their representation to reflect the accurate category (tube events). Again, the positive results in the postinteraction condition could not come from a perceptual preference for the changed (taller) object. If it were the case, the same results should have been obtained in the preinteraction condition. Moreover, the positive results in the postinteraction condition helped alleviate a potential concern that perhaps seeing a tube distracted infants, resulting in their overall disengagement from the event or inability to detect any changes. Here the infants saw a tube being rotated during the event but detected the change. However, it is logically possible that seeing a rotated tube could increase the cognitive load of the task at hand (without involving any consideration of the event category), resulting in infants' failure to detect the change especially when the tube is shown before the change. Recall that infants in Experiment 1 saw the rotated tube before the change. Infants' detection of the change in Experiment 1 speaks against the alternative interpretation, at least to some extent.

These findings are important for a number of reasons. First, they confirm the results of Experiment 2, demonstrating that prior exposure to a cover guided infants to determine the event as covering, allowing them to notice the height change. Second, the results suggest that infants are capable of updating their representation on the fly. After an event category has been selected, new information provided relatively early in the process can still be taken into account and override the initial category. Third, the timing of information matters. In the present case, new information was ignored if provided near the end of the process. Thus, depending on when it is received, categorical cues can exert strong influence or no influence at all on infants' representation of the event. Finally, the results suggest that infants attend closely to each component of the event and the order it appears, enabling them to notice new categorical cues and integrate the cues into their existing representation.

\section{General Discussion}

As demonstrated in a variety of looking-time and action tasks $[15,21,29]$, surprising decalages have been observed in infants' ability to use object information across different types of events. For example, infants succeed in using height information for one type of events (e.g., covering events) but fail to do so for another type of events (e.g., tube events) until a few months older. Building on these past findings, the present research examined the role of object interaction by manipulating its presence during an event (Experiment 1), replicated the effects of event categories with categorical cues presented before the event (Experiment 2), and investigated the effects of timing by showing infants conflicting cues at different segments of an event (Experiment 3). The results provide evidence that helps specify three important facets of the mechanism underlying infants' representations of physical events.

First, our findings support the notion that when infants watch an event that involves no object interaction, even if it provides some temporal and motion cues, they are less likely to take into account the type of events they are watching. But instead infants rely on their memory of objects to notice any changes to object features. In Experiment 1 , the 12-month-olds watched a tube event in which the tube moved independently of the object. Even though existing work has converged to show that infants this age tend to miss changes to object height in tube events, the 12-montholds in Experiment 1 had no difficulty noticing that the object changed from short to tall or vice versa. Thus, witnessing the interaction is critical to classifying the event and to having event-category effects on infants' use of object information.

Second, our findings show that categorical cues presented before the event will lead infants to perceive the event as such. Consequently, perceptually identical events can be viewed as different if different categorical cues are provided beforehand. In Experiment 2, the 12-month-olds detected the change in object height if they were shown, prior to the event, a cover but failed to do so if they were shown a tube. Thus, the infants perceived the same change in the same event differently depending on the categorical cue provided to them several seconds ago, suggesting that the cue may be held in memory and gets used at the point of interaction.

Finally, our findings help clarify the fine-grained, temporal aspect of the representational process. Although categorical cues can influence how infants view an event, the cues will cease to affect infants' representations of physical events if they are provided after object interaction - too late in the process. In Experiment 3, the 12-month-olds were guided by the cue provided before the event to believe that a cover was being used in the event. This representation could be overridden by seeing a tube before it was lowered over the object. The category of the current event was updated from covering to tube, and as such infants failed to notice the change in object height just as those who saw tube events in the previous studies. In contrast, if infants were shown the tube after it had interacted with the object, they seemed to maintain the initial category (covering), which allowed them to notice the change.

Together, the present findings lend support for the theoretical account summarized in Introduction, suggesting that information about object features such as height can be maintained in representations over time, but information 
about event categories may affect whether the information gets used or not (for parallel research in adults, e.g., [3840]). More broadly, the findings provide new evidence that infants, like older children and adults, actively construct the events they observe [41]. Whether they notice an implausible event depends on infants' current representation of the event.

So far, event-category effects have been observed mostly on infants' use of height information-a form feature. However, object features include not only form features but also surface features. Form features such as size and shape often determine the outcomes of object interaction (e.g., can a toy fit inside a box?), whereas surface features such as color and luminance often do not. It has been shown that infants are better at tracking color information if color predicts the type of action to be performed on an object [42], suggesting that the predictive power of the object feature matters. Research so far has primarily compared infants' use of form and surface information within occlusion events (e.g., [33, 42-45]) but not across event categories. The present research could be extended to surface features and manipulate the predictive power to test its role in event categorization.

Finally, infants' everyday observation of physical events often involves seeing an object move in relation to a stationary object. For example, a ball rolls behind a toy car and becomes fully hidden, or a toy car rolls to the front of a ball and fully hides it. In these examples, the figure (moving object) and reference (stationary object) are switched even though the same outcome is observed. The distinctions between figure and reference in object role are particularly important in linguistic processes as they determine how events are described [46]. Recent research has revealed that preverbal infants attend to figure/reference distinctions, which affects how they represent a dynamic or motion event (e.g., [46], for a review). In the present experiments, infants saw that a device moved to hide a stationary object. Will a switch in event role (i.e., figure-reference switch) affect infants' change detection? Will infants remain equally capable of tracking changes to the object if it serves as the figure that moves, rather than the reference that remains stationary as was shown in the present experiments? Answers to these questions will provide exciting possibilities for investigating the relations between linguistic structures and intuitive theories of physics.

To conclude, the present findings provide evidence that advances our understanding about the process by which infants represent physical events. Our findings highlight the role of object interaction in infants' representations of physical events; without this component, event categories cease to influence infants' use of object information. The present results also underscore the importance of timing of categorical cues and show that whether infants are affected by a cue will depend on when they receive it. The present findings thus clarify the origins of the decalages observed in infants' use of object information across different types of events and provide new evidence that infants actively construct and update their representations of physical events based on the ongoing flow of information about the events.

\section{Competing Interests}

The authors declare that there are no competing interests regarding the publication of this article.

\section{Acknowledgments}

This research was supported by the Faculty Research Grant from the Committee of Research at UCSC and by the Chiang Ching-kuo Foundation. The authors thank the staff of the Infant Development Lab at UCSC for their help with the data collection, Lili Beggs and Maggie Muir of the Sutter Lactation Center at Santa Cruz for their help with the participant recruitment, and the parents and infants who kindly participated in the research.

\section{References}

[1] R. Baillargeon, "The acquisition of physical knowledge in infancy: a summary in eight lessons," in Blackwell Handbook of Childhood Cognitive Development, U. Goswami, Ed., pp. 48-83, Blackwell, Malden, Mass, USA, 2002.

[2] S. Carey and F. Xu, "Infants' knowledge of objects: beyond object files and object tracking," Cognition, vol. 80, no. 1-2, pp. 179-213, 2001.

[3] E. S. Spelke, K. Breinlinger, J. Macomber, and K. Jacobson, “Origins of Knowledge," Psychological Review, vol. 99, no. 4, pp. 605-632, 1992.

[4] A. Aguiar and R. Baillargeon, "Developments in young infants' reasoning about occluded objects," Cognitive Psychology, vol. 45, no. 2, pp. 267-336, 2002.

[5] M. H. Bornstein, C. Mash, and M. E. Arterberry, "Young infants' eye movements over 'natural' scenes and 'experimental' scenes," Infant Behavior and Development, vol. 34, no. 1, pp. 206-210, 2011.

[6] M. H. Bornstein, C. Mash, and M. E. Arterberry, "Perception of object-context relations: eye-movement analyses in infants and adults," Developmental Psychology, vol. 47, no. 2, pp. 364-375, 2011.

[7] S. Denison and F. Xu, "Integrating physical constraints in statistical inference by 11-month-old infants," Cognitive Science: A Multidisciplinary Journal, vol. 34, no. 5, pp. 885-908, 2010.

[8] S. Denison and F. Xu, "Twelve- to 14-month-old infants can predict single-event probability with large set sizes," Developmental Science, vol. 13, no. 5, pp. 798-803, 2010.

[9] Y. Luo and R. Baillargeon, "When the ordinary seems unexpected: evidence for incremental physical knowledge in young infants," Cognition, vol. 95, no. 3, pp. 297-328, 2005.

[10] E. Spelke, "Initial knowledge: six suggestions," Cognition, vol. 50, no. 1-3, pp. 431-445, 1994.

[11] E. S. Spelke and S. J. Hespos, "Continuity, competence, and the object concept," in Language, Brain, and Cognitive Development: Essays in Honor of Jacques Mehler, E. Dupoux, Ed., pp. 325-340, The MIT Press, Cambridge, Mass, USA, 2001.

[12] S. J. Hespos and R. Baillargeon, "Infants' knowledge about occlusion and containment events: a surprising discrepancy," Psychological Science, vol. 12, no. 2, pp. 141-147, 2001.

[13] S. J. Hespos and R. Baillargeon, "Décalage in infants' knowledge about occlusion and containment events: converging evidence from action tasks," Cognition, vol. 99, no. 2, pp. B31-B41, 2006. 
[14] R. Baillargeon and J. DeVos, "Object permanence in young infants: further evidence," Child Development, vol. 62, no. 6, pp. 1227-1246, 1991.

[15] S.-H. Wang, R. Baillargeon, and S. Paterson, "Detecting continuity violations in infancy: a new account and new evidence from covering and tube events," Cognition, vol. 95, no. 2, pp. 129-173, 2005.

[16] S. M. Sitskoorn and A. W. Smitsman, "Infants' perception of dynamic relations between objects: passing through or support?" Developmental Psychology, vol. 31, no. 3, pp. 437-447, 1995.

[17] E. S. Spelke, "Core knowledge," American Psychologist, vol. 55, no. 11, pp. 1233-1243, 2000.

[18] G. L. Dueker and A. Needham, "Infants' object category formation and use: real-world context effects on category use in object processing," Visual Cognition, vol. 12, no. 6, pp. 11771198, 2005.

[19] A. Needham, "Infants' use of featural information in the segregation of stationary objects," Infant Behavior and Development, vol. 21, no. 1, pp. 47-76, 1998.

[20] A. Needham, G. Dueker, and G. Lockhead, "Infants' formation and use of categories to segregate objects," Cognition, vol. 94, no. 3, pp. 215-240, 2005.

[21] S.-H. Wang and R. Baillargeon, "Infants' physical knowledge affects their change detection," Developmental Science, vol. 9, no. 2, pp. 173-181, 2006.

[22] R. Baillargeon, M. Stavans, D. Wu et al., "Object individuation and physical reasoning in infancy: an integrative account," Language Learning and Development, vol. 8, no. 1, pp. 4-46, 2012.

[23] S.-H. Wang and R. Baillargeon, "Can infants be 'taught' to attend to a new physical variable in an event category? The case of height in covering events," Cognitive Psychology, vol. 56, no. 4, pp. 284-326, 2008.

[24] S.-H. Wang and R. Baillargeon, "Detecting impossible changes in infancy: a three-system account," Trends in Cognitive Sciences, vol. 12, no. 1, pp. 17-23, 2008.

[25] R. Baillargeon and S.-H. Wang, "Event categorization in infancy," Trends in Cognitive Sciences, vol. 6, no. 2, pp. 85-93, 2002.

[26] M. Casasola, "When less is more: how infants learn to form an abstract categorical representation of support," Child Development, vol. 76, no. 1, pp. 279-290, 2005.

[27] A. M. Leslie and S. Keeble, "Do six-month-old infants perceive causality?” Cognition, vol. 25, no. 3, pp. 265-288, 1987.

[28] J. Rigney and S.-H. Wang, "Delineating the boundaries of infants' spatial categories: the case of containment," Journal of Cognition and Development, vol. 16, no. 3, pp. 420-441, 2015.

[29] S.-H. Wang and L. Kohne, "Visual experience enhances infants' use of task-relevant information in an action task," Developmental Psychology, vol. 43, no. 6, pp. 1513-1522, 2007.

[30] L. Kotovsky and R. Baillargeon, "The development of calibration-based reasoning about collision events in young infants," Cognition, vol. 67, no. 3, pp. 311-351, 1998.

[31] A. Aguiar and R. Baillargeon, "2.5-month-old infants' reasoning about when objects should and should not be occluded," Cognitive Psychology, vol. 39, no. 2, pp. 116-157, 1999.

[32] S. J. Hespos and R. Baillargeon, "Young infants' actions reveal their developing knowledge of support variables: converging evidence for violation-of-expectation findings," Cognition, vol. 107, no. 1, pp. 304-316, 2008.
[33] T. Wilcox, "Object individuation: infants' use of shape, size, pattern, and color," Cognition, vol. 72, no. 2, pp. 125-166, 1999.

[34] S.-H. Wang and S. R. Mitroff, "Preserved visual representations despite change blindness in infants," Developmental Science, vol. 12, no. 5, pp. 681-687, 2009.

[35] S. Duffy, J. Huttenlocher, S. Levine, and R. Duffy, "How infants encode spatial extent," Infancy, vol. 8, no. 1, pp. 81-90, 2005.

[36] Z. Káldy and A. M. Leslie, "A memory span of one? Object identification in 6.5-month-old infants," Cognition, vol. 97, no. 2, pp. 153-177, 2005.

[37] S.-H. Wang, "Priming 4.5-month-old infants to use height information by enhancing retrieval," Developmental Psychology, vol. 47 , no. 1, pp. 26-38, 2011.

[38] A. Hollingworth, "Failures of retrieval and comparison constrain change detection in natural scenes," Journal of Experimental Psychology: Human Perception and Performance, vol. 29, no. 2, pp. 388-403, 2003.

[39] S. R. Mitroff, D. J. Simons, and D. T. J. Levin, "Nothing compares 2 views: change blindness can occur despite preserved access to the changed information," Perception \& Psychophysics, vol. 66, no. 8, pp. 1268-1281, 2004.

[40] B. Strickland and B. J. Scholl, "Visual perception involves eventtype representations: the case of containment versus occlusion," Journal of Experimental Psychology: General, vol. 144, no. 3, pp. 570-580, 2015.

[41] J. Piaget, The Construction of Reality in the Child, Basic Books, New York, NY, USA, 1954.

[42] Z. Káldy and E. Blaser, "How to compare apples and oranges: Infants' object identification tested with equally salient shape, luminance and color changes," Infancy, vol. 14, no. 2, pp. 222243, 2009.

[43] Z. Káldy and A. M. Leslie, "Identification of objects in 9month-old infants: integrating 'what' and 'where' information," Developmental Science, vol. 6, no. 3, pp. 360-373, 2003.

[44] R. J. Woods and T. Wilcox, "Infants' ability to use luminance information to individuate objects," Cognition, vol. 99, no. 2, pp. B43-B52, 2006.

[45] L. Talmy, "Lexicalization patterns: semantic structure in lexical forms," in Language Typology and Syntactic Description: Grammatical Categories and the Lexicon, T. Shopin, Ed., Cambridge University Press, Cambridge, UK, 1985.

[46] L. Wagner and L. Lakusta, "Using language to navigate the infant mind," Perspectives on Psychological Science, vol. 4, no. 2, pp. 177-184, 2009. 


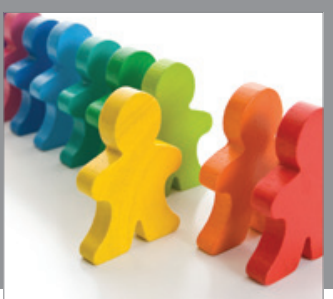

Autism

Research and Treatment
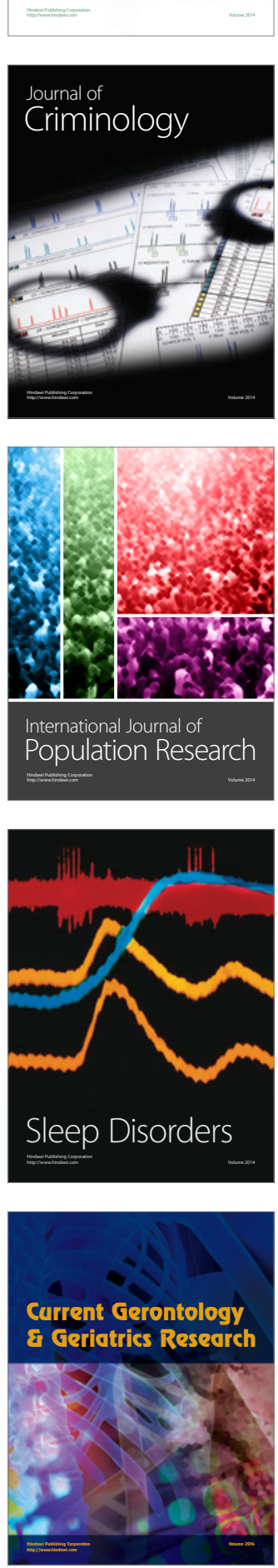

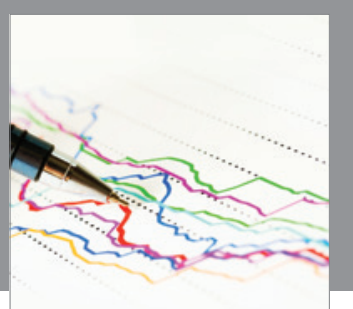

Economics

Research International
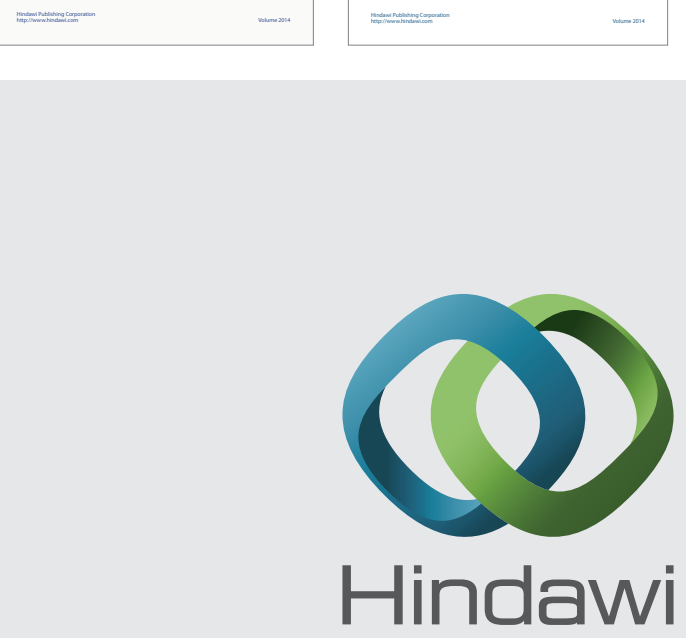

Submit your manuscripts at

http://www.hindawi.com
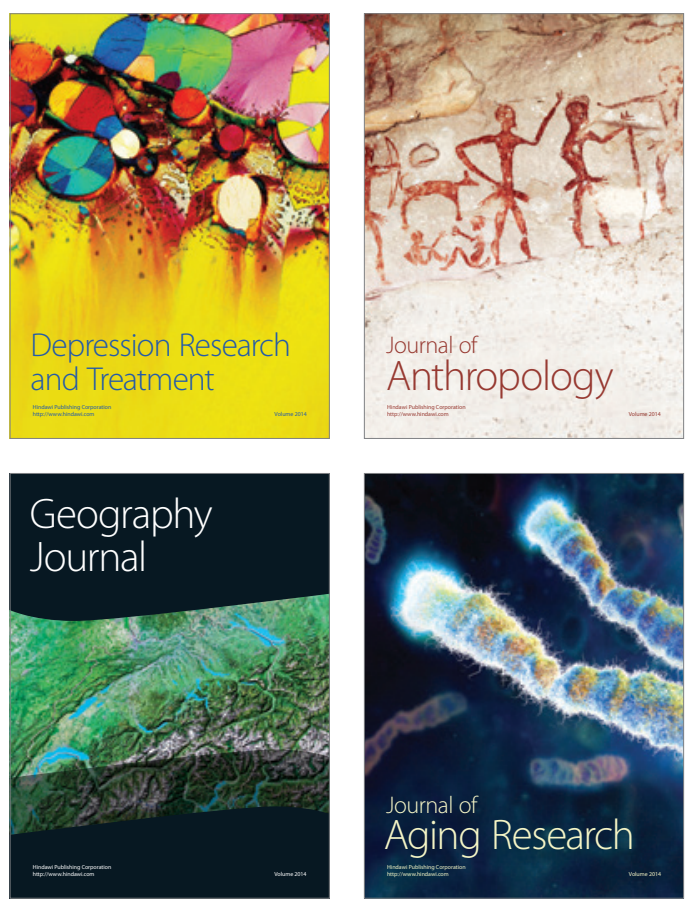
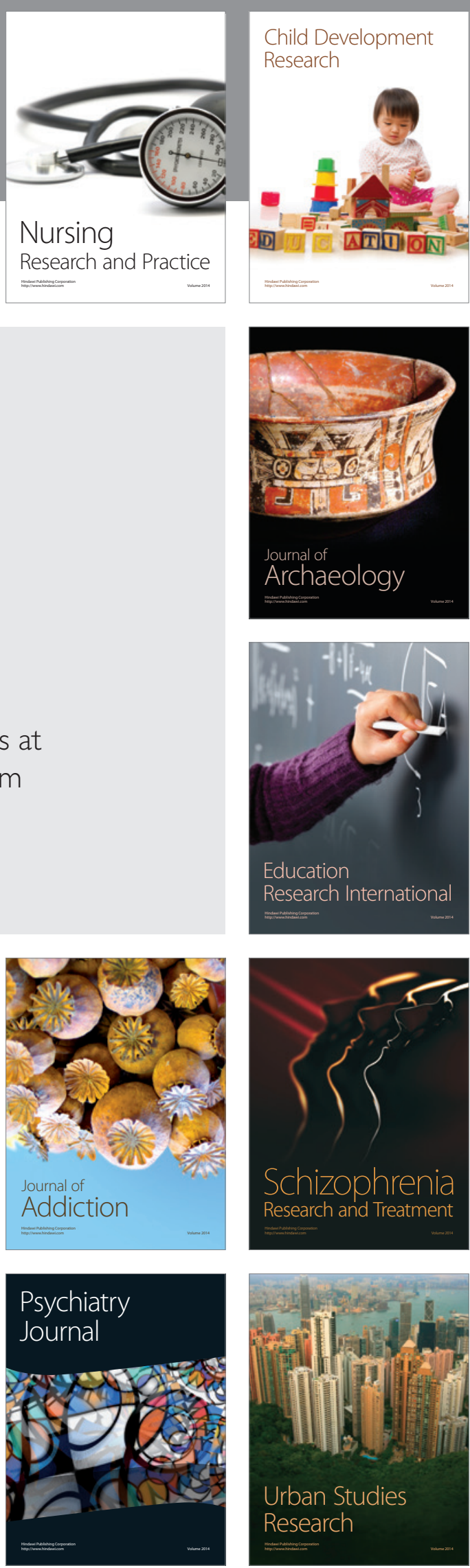